\title{
Diseño y puesta en marcha de un sistema de vigilancia epidemiológica en salud mental
}

\author{
Judith Scharager Goldenberg ${ }^{1}$ y Lorena Contreras Escudero ${ }^{2}$
}

RESUMEN Objetivos. Diseñar y poner a prueba un sistema de vigilancia que permita detectar y jerarquizar los problemas más relevantes de salud mental y establecer su asociación con las condiciones de vida de la comunidad estudiada.

Métodos. El sistema se diseñó y puso a prueba en un centro de salud de la comuna La Florida, región metropolitana de Chile. Los problemas de salud que se sometieron a vigilancia con seguimiento mensual fueron: adicción (al alcohol, tabaco, drogas y benzodiazepinas), violencia (violencia o sospecha de violencia intrafamiliar y abuso sexual) y trastornos en niños (déficit de atención y trastornos de conducta). Mediante un sistema computacional de información geográfica se combinaron estos elementos con datos sociodemográficos de las personas que acudieron a consulta y con datos territoriales que pueden considerarse factores protectores o de riesgo de los problemas de salud, y algunos de los cuales constituyen indicadores de las condiciones de vida de las personas.

Resultados. Se probó que el sistema de vigilancia epidemiológica propuesto - que incorpora las condiciones de vida y las variables ambientales - es una buena herramienta para la acción en salud y complementa la visión parcial de los sistemas de seguimiento, que no incluyen variables de contexto y se centran sólo en los problemas de salud. La información sintetizada en mapas permite visualizar simultáneamente diferentes capas de información sobre los factores relacionados con los problemas de salud estudiados, asociados a un territorio específico, con todas las variables ambientales que implican, en un formato de lectura ágil y evidente por sí mismo. Conclusiones. Estos resultados y la opinión convergente de los médicos participantes y usuarios permiten afirmar que el sistema de vigilancia epidemiológica puesto a prueba en fase experimental logró cumplir los requerimientos básicos de un método de esta naturaleza.

Palabras clave Vigilancia epidemiológica, salud mental, atención primaria, condiciones de vida.

Tradicionalmente, el análisis de los problemas de salud mental se ha llevado a cabo en el contexto de un para-

\footnotetext{
1 Escuela de Psicología, Pontificia Universidad Católica de Chile. La correspondencia debe ser dirigida a: Judith Scharager, Av. Vicuña Mackenna 4860, Santiago de Chile, Chile. Fax: 6864844. Correo electrónico: jscharag@puc.cl

2 Escuela de Psicología, Pontificia Universidad Católica de Chile, Santiago de Chile, Chile.
}

digma y criterios predominantemente clínicos, y solo en los últimos años se han reevaluado estos conceptos con un enfoque de salud pública. Afortunadamente, hoy en día es factible abordar estos problemas con tecnologías de salud que integran los aportes de las ciencias sociales y del comportamiento con la perspectiva de salud pública. Este nuevo enfoque y el cambio de perfil epidemiológico —en el que hoy predominan las enfermedades vinculadas a condiciones y estilos de vida- se presentan en Chile en un período en el que se han producido diversas innovaciones estructurales en el sector de la salud. Estos cambios, como la descentralización, la coordinación intersectorial, la estrategia de atención primaria y la priorización de problemas de salud pública del país, configuran un escenario propicio para 
desarrollar y evaluar herramientas de gestión en salud aplicadas al campo de la salud mental, las cuales constituyen una de las necesidades prioritarias.

El propósito central de este trabajo fue la prueba empírica de un sistema integral piloto de vigilancia epidemiológica en salud mental. Su diseño y puesta en marcha se fundamentó principalmente en el enfoque teórico desarrollado por P. L. Castellanos $(1,2)$ y otros investigadores (3-8), en el que se asigna a las condiciones de vida de las personas un papel fundamental en la determinación de los problemas de salud. Para efectos del análisis, las condiciones de vida pueden ser agrupadas en cuatro grandes dimensiones - que representan procesos interactivos-, cada una de las cuales se expresa predominantemente en un grupo de problemas de salud y se corresponde con un campo de respuestas sociales:

1. Dimensión biológica: Representa el potencial genético y la capacidad inmunológica. Esta dimensión se manifiesta en el ámbito de la concepción, gestación, nacimiento, desarrollo, desgaste y degeneración de las personas. Entre las expresiones colectivas más relevantes se pueden señalar la estructura por edades, la tasa de natalidad, el estado inmunitario colectivo, la frecuencia de características biológicas protectoras o de riesgo para algunas enfermedades transmisibles, etc.

2. Dimensión ambiental: Incluye los procesos predominantemente ecológicos del medio ambiente residencial y laboral. Esta dimensión tiene su expresión en las características climáticas y ambientales generales y en el espacio geográfico del lugar donde se inserta la comunidad en estudio, incluyendo las condiciones de saneamiento básico predominantes en el medio residencial y laboral del grupo. En definitiva, alude a la salud ambiental en un territorio específico.

3. Dimensión conductual: Incluye determinantes culturales, estilos de vida, hábitos y formas de conducta. La dinámica de esta dimensión se expresa tanto a nivel individual

FIGURA 1. Modelo de análisis de la situación de saluda

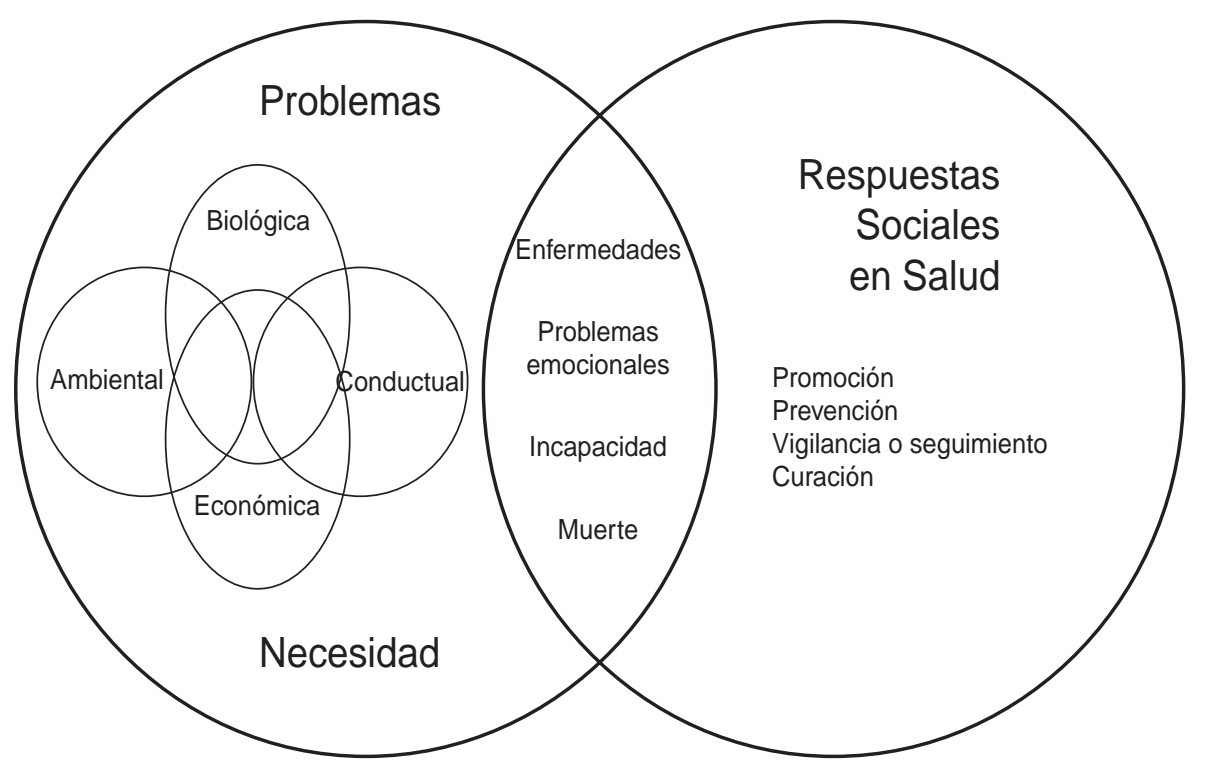

adaptado de P. Castellanos (3).

como colectivo, en procesos educativos formales e informales, formas organizadas o individuales de comportamiento frente a problemas y necesidades de salud. En este dominio, la participación y organización son expresiones predominantes.

4. Dimensión económica: Esta dimensión abarca aquellos procesos que se relacionan con la producción, distribución y consumo de bienes y servicios, y se expresa en los niveles de ingreso de los grupos familiares (2).

Para entender mejor el modelo de análisis de la situación de salud, este se ilustra en la figura 1. En ella se puede observar que las dimensiones no son excluyentes entre sí y se generan a partir del predominio de procesos en una dimensión dada en comparación con otra. En este sentido, se espera que existan interacciones entre ellas. Por otro lado, la dimensión conductual, tal como la desarrolla este modelo, puede resultar limitante por no considerar la visión más psicológica o psicosocial de la emergencia de los problemas de salud mental, tales como estrés, apoyo social, redes de apoyo y experiencias previas o concomitantes a estos problemas. No obstante, y aun cuando el modelo de Castellanos constituyó el fundamento teórico para la búsqueda y organización de la información necesaria en la construcción del modelo de vigilancia epidemiológica que aquí se presenta, resulta teóricamente insuficiente como fundamento para la comprensión de fenómenos de la salud mental y las condiciones de vida de las personas.

La interacción de los procesos involucrados en las dimensiones de las condiciones de vida del modelo presentado da origen a un perfil de necesidades y problemas de salud —que se corresponden a su vez con un perfil de acciones de salud-que caen en el ámbito de lo que se puede llamar "respuestas sociales". Se debe destacar que estas relaciones no son lineales ni determinísticas, sino más bien de carácter dinámico y probabilístico.

A partir de este modelo, cualquier estudio de las condiciones de vida de una comunidad debería identificar variables, expresadas en indicadores específicos, que den cuenta de cada una de las dimensiones descritas. En el cuadro 1 se presentan las empleadas en este estudio. 
CUADRO 1. Variables e indicadores de condiciones de vida empleados en el estudio

\begin{tabular}{|c|c|c|}
\hline \multirow[b]{2}{*}{ Dimensión } & \multicolumn{2}{|c|}{ Entorno } \\
\hline & Individual y familiar & Sociocomunitario \\
\hline Biológica & $\begin{array}{l}\text { Edad } \\
\text { Sexo } \\
\text { Problema de salud en la esfera so- } \\
\quad \text { mática }^{a}\end{array}$ & $\begin{array}{l}\text { Estructura de la comuna por edades } \\
\text { Distribución de la comuna por sexo }\end{array}$ \\
\hline Ambiental & $\begin{array}{l}\text { Calidad de la vivienda }\left(\mathrm{CAS}^{\mathrm{c}}\right) \\
\text { Abastecimiento agua potable (CAS) } \\
\text { Bienes confort habitacional (CAS) } \\
\text { Puntaje ficha CAS }\end{array}$ & $\begin{array}{l}\text { Áreas verdes }{ }^{b} \\
\text { Trazado vial }^{b} \\
\text { Densidad poblacional }\end{array}$ \\
\hline Conductual & $\begin{array}{l}\text { Problemas de salud mental (en la } \\
\quad \text { esfera relacional) })^{b}\end{array}$ & $\begin{array}{l}\text { Botillerías }^{b} \\
\text { Puntos de tráfico de drogas según } \\
\text { denuncias }{ }^{b} \\
\text { Clubes deportivos }^{b} \\
\text { Organizaciones comunitarias } \\
\text { Or }\end{array}$ \\
\hline Económica & $\begin{array}{l}\text { No se dispone de datos (Ficha CAS } \\
\text { incompleta en este item) }\end{array}$ & $\begin{array}{l}\text { Áreas de "bolsones" de pobreza } \\
\qquad(\mathrm{CAS}<500)^{\mathrm{b}}\end{array}$ \\
\hline
\end{tabular}

La recolección y organización de la información relativa a estas dimensiones se puede llevar a cabo mediante estrategias descritas en la literatura (1). En este estudio se propone la aplicación de un sistema de información geográfica (SIG) como técnica innovadora para el análisis de la situación de salud y de las condiciones de vida de una localidad determinada. Los SIG constituyen herramientas dinamizadoras del análisis y presentación de este tipo de información y toman debidamente en cuenta el territorio, que es un elemento a menudo olvidado en este tipo de análisis. La aplicación de un SIG permite elaborar informes que combinan la presentación gráfica de mapas sectorizados por alguna unidad geográfica de la zona, en los que se puede visualizar la distribución espacial de eventos, fenómenos, casos portadores de alguna enfermedad $u$ otro problema de salud, conjuntamente con indicadores de sus condiciones de vida. En resumen, un SIG puede ayudar a un equipo local de salud a reconocer y entender la heterogeneidad social y la variabilidad epidemiológica de la población bajo su responsabilidad. De esta manera, si se articula un
SIG con un buen sistema de información que permita el registro de problemas de salud y factores relacionados con las condiciones de vida, se puede concebir un sistema de vigilancia epidemiológica de dichos problemas, ubicado dentro de un contexto determinado y asociado con el entorno y las condiciones de vida de quienes padecen esos problemas (Toledo J, comunicación personal, 1996).

La puesta en marcha de un sistema de vigilancia asociado a cualquier estrategia de recolección de datos requiere de indicadores que permitan el seguimiento de los problemas de salud mental. Los indicadores epidemiológicos clásicos de mortalidad y morbilidad, sin embargo, no son los más apropiados. La mayoría de estos problemas tienen una letalidad baja - salvo en casos como el alcoholismo, en el que se puede establecer una medición indirecta por la tasa de mortalidad por cirrosis hepática, informes de suicidios o las muertes por violencia. Los casos de trastornos mentales, por otro lado, no requieren notificación obligatoria, por lo que su registro es poco frecuente y sistemático (9-12). Por consiguiente, se debe definir un conjunto alternativo de indicadores específicos, susceptibles de ser registrados periódica y sistemáticamente en el nivel primario por profesionales de la salud no especialistas en salud mental. No obstante, la selección de indicadores apropiados es una tarea compleja, pues como se observa en la literatura, existe una gran dispersión de estrategias empleadas en el nivel primario para la cuantificación de problemas de salud mental, y se utilizan instrumentos de distinto nivel de complejidad, orientados al tamizado o al diagnóstico - tales como entrevistas con procedimientos explícitos y estandarizados-, o a mediciones que ofrecen datos de riesgo estadístico y que requieren un largo entrenamiento y exigen mucho tiempo para su aplicación.

En este escenario, el objetivo del presente estudio fue diseñar y poner a prueba un sistema de vigilancia que contemplara la definición de indicadores adecuados, que permitiera detectar y jerarquizar problemas relevantes de salud mental y que permitiera establecer su asociación con las variaciones en las condiciones de vida de la comunidad estudiada. Para estos efectos se identificó la comuna La Florida, de la Región Metropolitana en la zona central de Chile, cuyas características permitían introducir innovaciones a un modelo tradicional de vigilancia en salud. En el momento del estudio, esta comuna era étnicamente homogénea y tenía una estructura poblacional joven de alrededor de 335000 habitantes. La comuna cuenta con cinco centros de atención primaria de salud con un nivel adecuado de informatización de registros. Esto permitió instalar el sistema en uno de los centros sin añadir costos al servicio ni hacer modificaciones sustantivas en su práctica habitual de atención o en los sistemas de información.

Se esperaba que el sistema cumpliera con las siguientes características:

- que fuera un sistema de detección y registro continuo simple y operativo, capaz de detectar la aparición de ciertos problemas de salud mental y los cambios en su incidencia;

- que su diseño permitiera estudiar las posibles asociaciones entre distintas configuraciones de las condi- 
ciones de vida y los cambios en la salud de las personas, en un espacio geográfico acotado y conocido;

- que fuera capaz de generar información útil y de sintetizar distintos tipos de datos en una imagen, para orientar la gestión en salud y sugerir líneas de acción en la oferta de servicios, la asignación de recursos y el establecimiento de prioridades en el nivel local.

\section{MATERIALES Y MÉTODO}

En reuniones con el equipo de médicos de los programas del Adulto y del Niño ${ }^{3}$ se seleccionaron los problemas de salud que se someterían a vigilancia y se establecieron los criterios para su pesquisa y registro. Los propios médicos, a partir de su experiencia clínica, seleccionaron los problemas de salud mental que estimaban más relevantes y definieron los códigos que se utilizarían para su entrada al sistema INFOSAL (sistema de información computarizado instalado en los centros de salud de La Florida), y se escogieron aquellos que resultaban más fáciles de recordar y anotar.

Con el objetivo de homogeneizar los criterios, uno de los médicos del centro de salud, que terminaba su formación de postítulo en medicina familiar, realizó un taller para sus pares, en el que se compartieron y acordaron los criterios diagnósticos de los problemas de salud mental identificados para someter a vigilancia, basados en el Manual diagnóstico y estadístico de trastornos mentales (DSMV-IV) (13).

Los médicos consignaron, con un código ad hoc, los problemas de salud mental detectados en los formularios de registro diario que habitualmente se usan para consignar los casos atendidos. Esta información era posteriormente introducida en el compu-

\footnotetext{
Estos programas forman parte de las estrategias del Ministerio de Salud de Chile para la atención primaria. Es importante destacar que los programas del Adulto y del Niño abarcan la mayor parte de la población, a diferencia de aquellos que responden a problemas de salud más puntuales.
}

CUADRO 2. Registros de frecuencias y porcentajes mensuales de problemas de salud agrupados en categorías durante el período completo de vigilancia

\begin{tabular}{|c|c|c|c|c|c|c|c|c|}
\hline \multirow[b]{2}{*}{ Mes } & \multicolumn{2}{|c|}{$\begin{array}{l}\text { Trastornos } \\
\text { somáticos }\end{array}$} & \multicolumn{2}{|c|}{ Adicciones } & \multicolumn{2}{|c|}{$\begin{array}{l}\text { Violencia } \\
\text { intrafamiliar }\end{array}$} & \multicolumn{2}{|c|}{$\begin{array}{c}\text { Trastornos en } \\
\text { niños }\end{array}$} \\
\hline & No. & $\%$ & No. & $\%$ & No. & $\%$ & No. & $\%$ \\
\hline Septiembre & 1221 & 94,5 & 64 & 5,05 & 3 & 0,23 & 4 & 0,31 \\
\hline Octubre & 1333 & 95,8 & 25 & 1,8 & 14 & 0,95 & 19 & 1,37 \\
\hline Noviembre & 849 & 94,4 & 34 & 3,75 & 6 & 0,67 & 10 & 1,11 \\
\hline Diciembre & 582 & 94,9 & 16 & 2,61 & 5 & 0,82 & 10 & 1,63 \\
\hline Enero & 1370 & 96,4 & 36 & 2,53 & 8 & 0,56 & 7 & 0,49 \\
\hline Febrero & 1054 & 98,3 & 13 & 1,21 & 2 & 0,19 & 3 & 0,28 \\
\hline Marzo & 1228 & 97,5 & 17 & 1,35 & 3 & 0,24 & 11 & 0,87 \\
\hline
\end{tabular}

tador por un funcionario del SOME, ${ }^{4}$ quien además producía los informes mensuales con las estadísticas de atención y sus correspondientes categorías diagnósticas.

Paralelamente se activó el sistema de información geográfica, el cual ya existía en los centros de salud de nivel primario de la comuna La Florida, y se generaron las bases de datos del trazado de calles de la zona de estudio, de los límites del área, y de las unidades vecinales y otras entidades de menor espacio geográfico.

A partir de estos antecedentes se elaboraron informes mensuales parciales de los resultados del sistema de vigilancia. Estos informes se generaron con los datos que habitualmente brinda INFOSAL, incluidos los registros de algunos índices sociodemográficos de los pacientes con problemas de salud mental, combinados con la información geográfica. Como resultado, se obtuvieron mapas con la distribución en el territorio de los casos atendidos. Estos mapas permitían visualizar al mismo tiempo -en una "capa" común- el territorio vinculado al centro de salud y la localización de los domicilios de los casos, y fueron distribuidos periódicamente a los médicos como una retroalimentación del funcionamiento del sistema.

Finalmente, se pidió a todos los médicos participantes que evaluaran el

\footnotetext{
4 SOME: Servicio de Orientación Médico-Estadístico
}

sistema de vigilancia propuesto basándose en una pauta diseñada al efecto.

\section{RESULTADOS Y DISCUSIÓN}

Los problemas de salud que se ingresaron al sistema computacional INFOSAL fueron los siguientes:

- Adicción al alcohol

- Adicción al tabaco

- Abuso de benzodiazepinas

- Adicción a drogas

- Sospecha de violencia intrafamiliar

- Violencia intrafamiliar

- Abuso sexual

- Déficit de atención en niños

- Trastorno de conducta en niños

- Trastornos somáticos (ajenos a las categorías anteriores)

Dado que hubo un bajo porcentaje de registros de algunos de los problemas estudiados, se decidió agruparlos en categorías más amplias que permitieran visualizar mejor su evolución en el tiempo. Las tres categorías son:

- Adicciones: adicción al alcohol, tabaco, drogas y benzodiazepinas

- Violencia: violencia intrafamiliar, sospecha de violencia intrafamiliar y abuso sexual

- Trastornos en niños: déficit de atención y trastornos de conducta

En el cuadro 2 se muestra una síntesis de la frecuencia y el porcentaje del registro de estas categorías de proble- 
FIGURA 2. Evolución de problemas de salud mental agrupados en categorías

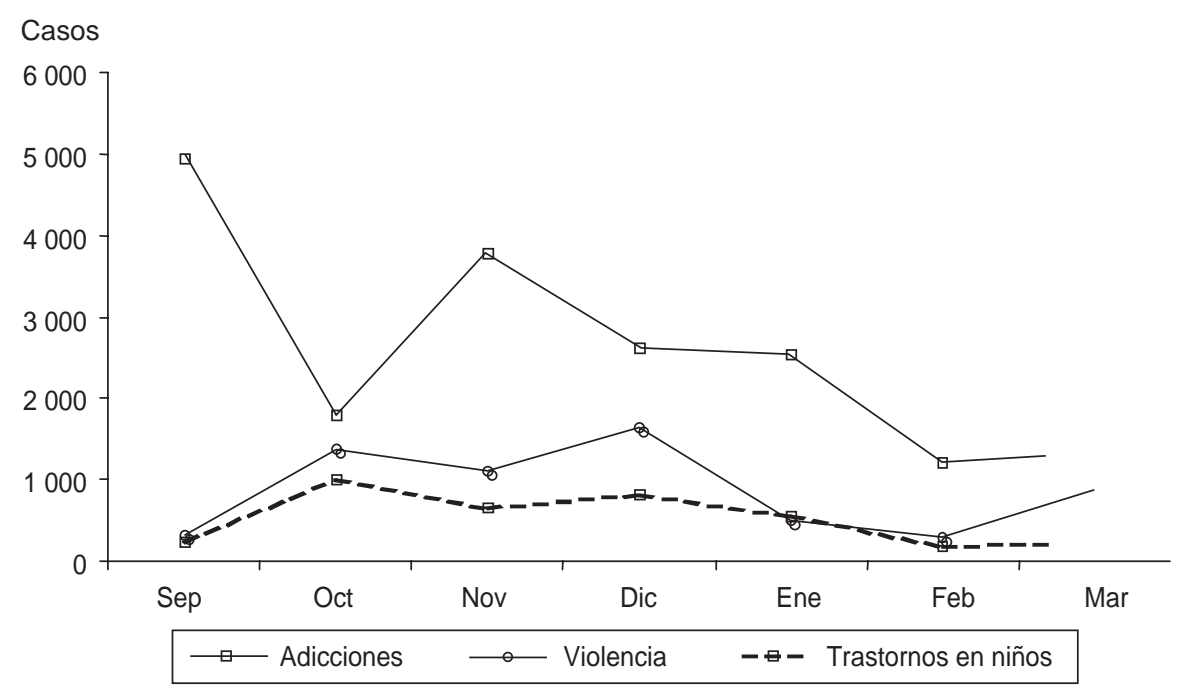

mas de salud durante los siete meses que funcionó este sistema de vigilancia. Como se observa, algunos de estos resultados podrían ser atribuibles a tendencias estacionales. Por ejemplo, un mayor porcentaje de consultas en las que se detectan problemas de adicción durante septiembre, que es el mes en que aumenta el consumo de alcohol por motivo de las festividades nacionales, y una disminución de diagnósticos relacionados con problemas de los niños en meses de vacaciones escolares de verano. Por otro lado, es importante destacar que, por primera vez, los médicos registraron problemas de violencia o abuso sexual en los formularios de atención diaria $\mathrm{y}$, aunque la frecuencia era baja, el registro se mantuvo a lo largo de todo el período de prueba del sistema de vigilancia. En este sentido, es importante señalar que este sistema de vigilancia se puso en marcha solo a un mes de la promulgación de la Ley de Violencia Intrafamiliar, publicada en el Diario Oficial. ${ }^{5}$

$\mathrm{Al}$ representar estos resultados gráficamente (figura 2), se puede observar que los ligeros aumentos en las pes-

\footnotetext{
5 Ley 19.325: Esta ley establece normas sobre procedimientos y sanciones relativas a los actos de violencia intrafamiliar. Tipifica los actos de violencia intrafamiliar y señala las sanciones y el procedimiento aplicable al efecto.
}

quisas de violencia intrafamiliar se manifiestan simultáneamente con aumentos en las frecuencias de problemas de conducta en los niños (no necesariamente en los mismos niños) en los meses en los que suele haber evaluaciones del rendimiento académico en los establecimientos educacionales chilenos.

En el cuadro 3 se presenta el registro acumulado durante todo el período de observación de los problemas de salud mental y su relación porcentual con el total de afecciones atendidas por los médicos del centro de salud. Los porcentajes de registro de los problemas de salud mental estuvieron muy por debajo del nivel obtenido en otros estudios epidemiológicos (14); no obstante, debe tomarse en consideración que en el presente estudio no se utilizó un instrumento diagnóstico o de tamizado específico, sino solo la observación clínica de médicos no especialistas, previo acuerdo de los criterios a aplicar para tal efecto. Es probable que si se aplicaran instrumentos estandarizados en un estudio orientado específicamente a su pesquisa, se obtendrían valores de prevalencia más elevados. Por otro lado, no se debe olvidar que los médicos pesquisaron los problemas de salud mental durante las consultas de atención o control de salud, al mismo tiempo que prestaban el servicio de atención solicitado, en un lapso no mayor de 10 minutos, que es el tiempo habitual de atención a un paciente en estos centros.

Se hicieron, además, comparaciones de los distintos problemas registrados por género y grupos de edad. Los datos de frecuencia acumulada en el período de observación por grupos de problemas, diferenciados por sexo y grupos de edad, se presentan en los cuadros 4 y 5 , respectivamente.

En el cuadro 4 se advierte un mayor registro de problemas de salud mental en la población masculina, salvo en el caso de los problemas relacionados con la violencia intrafamiliar, donde hay un predominio de registro de mujeres. Al incluir la variable edad, se incrementan los registros en la población adulta, con predominio de los problemas de adicción (cuadro 5).

Por otro lado, el uso del SIG permitió desplegar en un mismo mapa geográfico diversos aspectos de los problemas de salud que se estaban investigando. Los médicos que participaron en este sistema de vigilancia recibieron informes periódicos que in-
CUADRO 3. Problemas de salud mental (agrupados en categorías) y somáticos investigados durante todo el período de observación

\begin{tabular}{lcc}
\hline \multicolumn{1}{c}{ Problema } & Número de notificaciones & Porcentaje \\
\hline De salud mental & 313 & 3,93 \\
Adicciones & 205 & 2,57 \\
Trastornos en niños & 67 & 0,84 \\
Violencia intrafamiliar & 41 & 0,52 \\
Somáticos & 7651 & 96,07 \\
Total & 7964 & 100,0 \\
\hline
\end{tabular}


CUADRO 4. Problemas de salud mental (agrupados por categorías) y somáticos, según sexo. En paréntesis aparece el porcentaje con respecto al número total de problemas de salud notificados

\begin{tabular}{lrrr}
\hline & \multicolumn{2}{c}{ Sexo } & \\
\cline { 2 - 3 } Problemas de salud & Femenino & Masculino & Total \\
\hline De salud mental & $120(1,6)$ & $189(2,4)$ & $309(4,0)$ \\
Adicciones & $67(0,9)$ & $134(1,7)$ & $201(2,6)$ \\
Trastornos en niños & $21(0,3)$ & $46(0,6)$ & $67(0,9)$ \\
Violencia intrafamiliar & $32(0,4)$ & $9(0,1)$ & $41(0,5)$ \\
Trastornos somáticos & $4484(57,8)$ & $2966(38,2)$ & $7450(96,0)$ \\
$\quad$ Total & $4604(59,3)$ & $3155(40,7)$ & $7759(100,0)$ \\
\hline
\end{tabular}

CUADRO 5. Problemas de salud mental (agrupados por categorías) y somáticos, según grupos de edad. En paréntesis aparece el porcentaje con respecto al número total de problemas de salud reportados

\begin{tabular}{lcrr}
\hline & \multicolumn{2}{c}{ Grupo de edad } & \\
\cline { 2 - 3 } Problemas de salud & \multicolumn{1}{c}{ Niños } & Adultos & Total \\
\hline De salud mental & $78(1,0)$ & $235(2,9)$ & $313(3,9)$ \\
Adicciones & $1(0,01)$ & $204(2,6)$ & $205(2,6)$ \\
Trastornos en niños & $67(0,8)$ & $0(0,0)$ & $67(0,8)$ \\
Violencia intrafamiliar & $10(0,1)$ & $31(0,4)$ & $41(0,5)$ \\
Trastornos somáticos & $3284(41,3)$ & $4360(54,8)$ & $7644(96,1)$ \\
$\quad$ Total & $3362(42,3)$ & $4595(57,7)$ & $7957(100,0)$ \\
\hline
\end{tabular}

cluían tablas y mapas impresos. A modo de ilustración se incluyen tres de esos mapas. En el de la figura 3 es posible visualizar la distribución de la población que se encuentra bajo la línea de pobreza, según la ficha CAS, ${ }^{6}$ en el área de asignación del centro de salud Los Castaños, por unidades vecinales. En la figura 4 se presenta la distribución de los casos de adultos con problemas de adicción al alcohol pesquisados durante todo el período que duró la aplicación piloto de este sistema de vigilancia, y su localización en las distintas unidades vecinales de la población asignada al centro de salud Los Castaños.

$\mathrm{Al}$ observar los mapas surgen algunas hipótesis respecto a los problemas

\footnotetext{
6 Sistema de Clasificación Socioeocómica utilizado en los centros de salud chilenos.
}

de adicción al alcohol y su relación con su ubicación geográfica y entorno. Por ejemplo, se podría considerar la existencia de una relación con la cercanía a lugares de expendio de bebidas alcohólicas o puntos de tráfico y consumo de drogas ilegales - como se aprecia en la figura 5-, con bolsones de pobreza - como se observa en la figura 3-, con la densidad poblacional o con otra variable que parezca pertinente.

Es importante destacar, por otro lado, que la evaluación que se puede hacer de la utilidad de la representación gráfica en mapas con distintas capas de información y los problemas de salud mejora considerablemente si se hace con un computador y el programa correspondiente, ya que permite alternar los planos de datos y probar hipótesis de asociación en forma interactiva y dinámica. Esta posibilidad de combinar diversas capas de datos es particularmente importante, ya que la observación conjunta de solo dos capas de datos puede llevar a hipótesis equivocadas. Es así como, por ejemplo, pareciera que los casos de alcoholismo se concentraran en algunas unidades vecinales, lo que podría indicar alguna tendencia por efecto de las características del entorno. Sin embargo, si se analiza la distribución de los domicilios de todas las demás personas que acudieron a consulta (independientemente de su diagnóstico), se advierte que tienen la misma distribución, lo que presumiblemente se relaciona con la densidad poblacional, de modo que la concentración de los casos de alcoholismo detectados en cada unidad vecinal resulta proporcional a la concentración o densidad poblacional y no es efecto de otra variable específica.

Finalmente, las opiniones de los médicos que participaron en el estudio complementaron las conclusiones derivadas de la lectura de las tablas y mapas, mediante un cuestionario diseñado especialmente para evaluar la factibilidad del sistema que fue aplicado al cuarto mes de uso. En sus respuestas, los médicos participantes señalaron varias ventajas del sistema, como el hecho de que ofrece información gráfica y rápida de la distribución de las enfermedades, permite detectar focos de problemas, registra la incidencia de enfermedades por zonas geográficas, ayuda a planificar los servicios de salud y a determinar los sectores de mayor riesgo, muestra dónde se concentran los pacientes con determinadas enfermedades, permite relacionar los trastornos de salud con el medio ambiente, entre otros. No se recibieron criterios negativos de parte de los médicos que efectuaron la evaluación, aunque señalaron que sería importante incorporar otras enfermedades al sistema de vigilancia y "relacionar enfermedad con medio ambiente".

A partir de la información sintetizada en las tablas, gráficos y mapas, y de la opinión convergente de los médicos participantes y usuarios, se puede afirmar que el sistema de vigilancia puesto a prueba en fase experimental logró cumplir los requerimientos 
FIGURA 3. Distribución porcentual de la población ubicada por debajo de la línea de pobreza (menos de 500 puntos según la ficha $\mathrm{CAS}^{\mathrm{a}}$ ), por unidades vecinales del área de atención del centro de salud Los Castaños

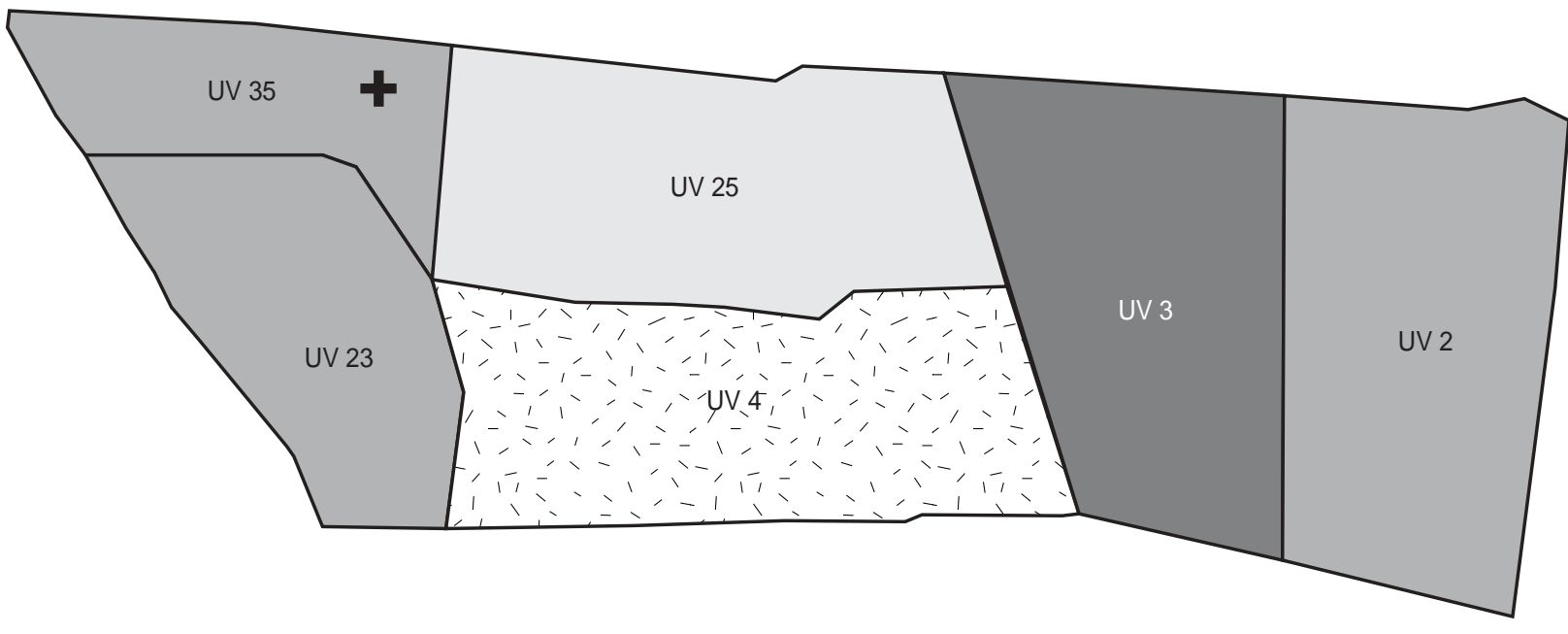

Límites entre las unidades vecinales
Localización del consultorio

Población (\%) con puntaje CAS menor de 500 puntos

$\square$ De 1 a $4 \% \quad \square$ De 5 a $8 \% \quad \square$ De 9 a $12 \% \quad \square$ De 13 a $17 \%$

Fuente: Dirección de Desarrollo Comunitario, Comuna de La Florida, 1996.

a CAS: Sistema de Clasificación Socioeconomica utilizada en los centros de salud chilenos.

FIGURA 4. Distribución de los casos de adultos con problemas de adicción al alcohol pesquisados durante el estudio, por unidades vecinales del área de atención del centro de salud Los Castaños

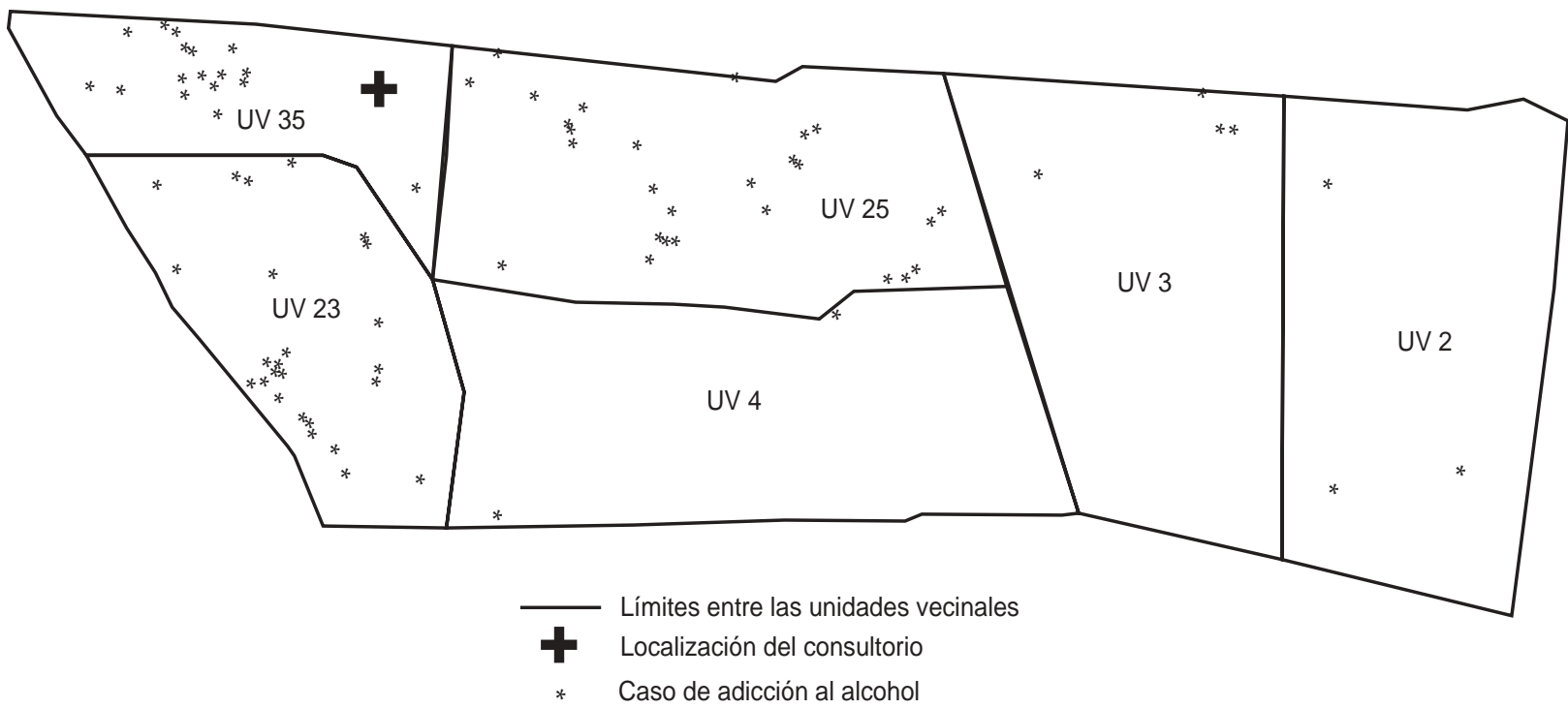

Fuente: Registro de estadística diaria de médicos generales. Consultorio Los Castaños. Consultas de septiembre de 1995 a marzo de 1996. 
FIGURA 5. Localización de sectores de tráfico y consumo de sustancias ilegales en el área de atención del centro de salud Los Castaños

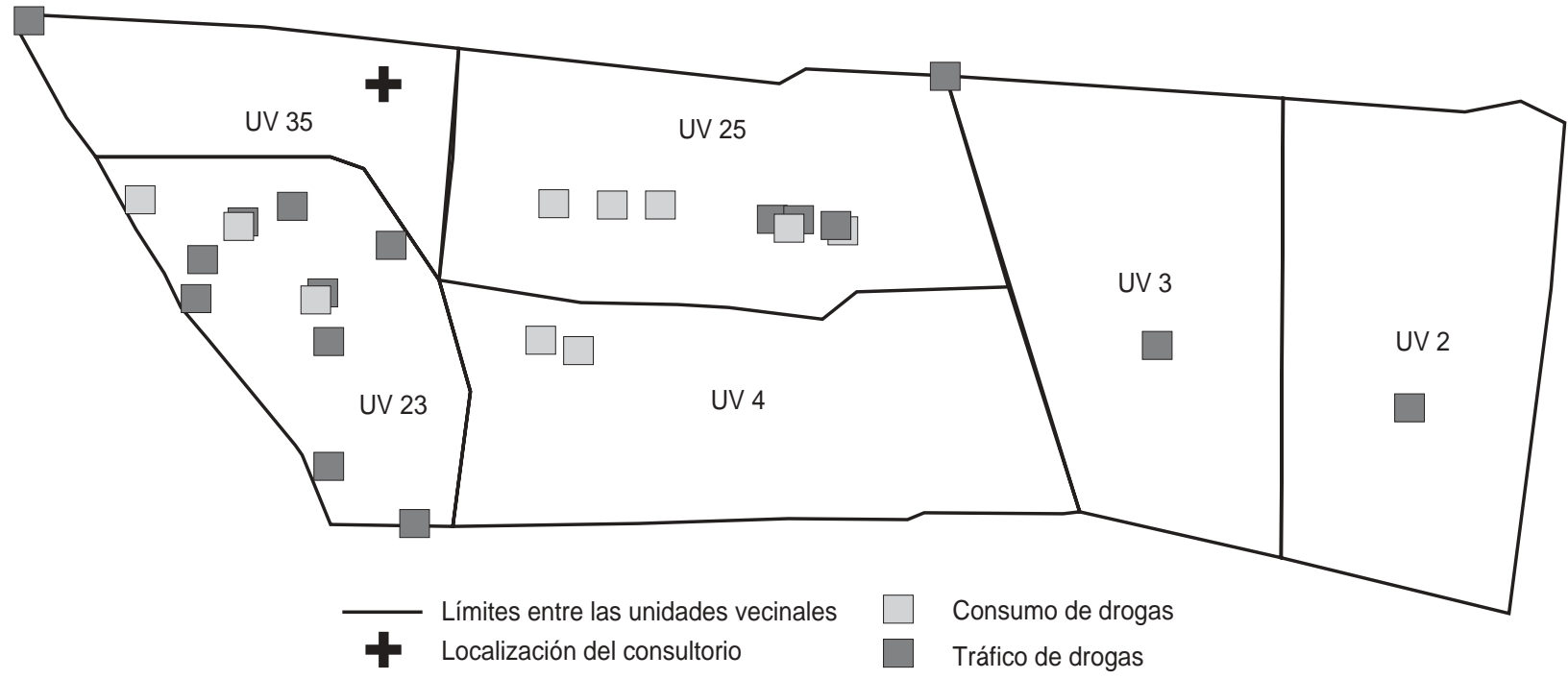

Fuente: Dirección de Desarrollo Comunitario. Comuna La Florida, 1996.

básicos de un método de esta naturaleza, ya que:

- permitió identificar y jerarquizar periódicamente los principales problemas de salud mental del área de atención del centro de salud Los Castaños, de acuerdo con la delimitación de áreas específicas preseleccionadas y los problemas prioritarios definidos por los usuarios del sistema;

- integró un sistema de apoyo permanente a la toma de decisiones, con retroalimentación oportuna para los encargados de la atención de salud, en un formato que facilita la lectura y comprensión de la información y que incorpora el medio ambiente como una variable;

- su implementación se ajusta a la dotación de los recursos humanos, materiales y económicos disponibles localmente y complementa los sistemas de registro de información en uso.

El análisis de la información obtenida en este estudio permite afirmar que la aplicación de este sistema de vigilancia epidemiológica en su versión piloto fue exitosa, por los siguientes criterios:

- El diseño e implementación del sistema de vigilancia propuesto fue coherente con las políticas nacionales y locales de salud mental.

- Su implementación no provocó gastos adicionales ni exigió tiempo adicional para su aplicación y solo requirió una breve capacitación, orientada fundamentalmente a la homogeneización de criterios en el reconocimiento de problemas de salud mental.

- Se pudieron elaborar perfiles de algunos problemas de salud mental de la comunidad en estudio — según grupos de edad, sexo y condiciones de vida- por unidad de espaciopoblación (unidad vecinal), y la incorporación del medio ambiente como variable.

- Permitió establecer asociaciones entre los perfiles de los problemas investigados y las condiciones de vida en las unidades espacio-población en estudio, segmentados por variables sociodemográficas.

- El sistema de vigilancia propuesto generó informes sencillos, acotados, con información numérica y gráfica, lo que, de acuerdo a la evaluación hecha por los profesionales de la salud que participaron, constituyó una información relevante y útil. La incorporación de esta innovación no interfirió con la práctica habitual de trabajo del centro de salud. Su aplicación se llevó a cabo sin incurrir en cambios tecnológicos que produjeran una resistencia al cambio por parte del personal asistencial ni de los directivos del centro.

- Fue accesible para los profesionales que requerían la información que genera este sistema de vigilancia epidemiológica, y permitió ampliar el espectro de problemas a investigar de acuerdo con las necesidades percibidas por los usuarios.

- Este sistema de vigilancia tuvo una alta aceptación por parte de los profesionales de la salud del centro, no solo por sus resultados, sino porque la estrategia empleada para su aplicación fue desarrollada tomando en consideración sus experiencias y aportes. Esto dio como resultado la validación de estos profesionales como actores relevantes en la generación de este sistema de informa- 
ción, lo que facilitó su incorporación a la práctica asistencial establecida.

En síntesis, se probó que el sistema de vigilancia epidemiológica propuesto, que incorpora las condiciones de vida y la variable ambiental, es una buena herramienta para la acción en salud, que complementa la visión parcial de los sistemas de seguimiento que no incluyen variables de contexto y se centran solo en los problemas de salud.

Un enfoque que integra los factores asociados a los problemas de salud, como el utilizado en el presente estudio, tiene una serie de ventajas. En primer lugar, permite evaluar cuán adecuada es la distribución de recursos -particularmente en la gestión local de salud-, ya que hace posible contrastar el ajuste entre las necesidades de prestaciones según las condiciones de vida de quienes presentan determinados problemas de salud y la distribución de estos recursos que se hace en un momento determinado. Asimismo, permite analizar inequidades y ajustar la distribución de recursos, con lo que potencia el trabajo intersectorial (salud y educación, por ejemplo) y facilita el diseño de actividades coherentes con las necesidades de la población estudiada. Por ejemplo, la focalización de las visitas domiciliarias de profesionales de la salud para la prevención del consumo de drogas o alcohol hacia aquellas zonas donde existe mayor concentración de casos y focos de venta y distribución de drogas. Esto, además, es más fácil de lograr con eficiencia mediante el formato de presentación de la información con gráficos y mapas, lo que complementa el despliegue cuantitativo de los datos. De este modo, exige menos tiempo para su interpretación $\mathrm{y}$ análisis por parte de los médicos o administradores de recursos para la salud.

Por otra parte, el enfoque empleado para el análisis de la situación sanitaria de un grupo o población debe reconocer los distintos niveles de información que dan cuenta de interacciones entre los atributos individuales y las relaciones sociales que se producen en un es- pacio poblacional, lo que genera una variedad de percepciones y consecuentes necesidades de salud. Si se opta por esta visión, se hace evidente que no es posible emplear un solo tipo de método de recolección y análisis de la información, independientemente del propósito. Ningún método es suficiente por sí solo para dar cuenta cabal de un fenómeno o proceso, ya que cada método revela diferentes aristas de la realidad empírica.

Por último, tal como se demostró en este estudio, existe aun otro nivel de análisis que amplía la perspectiva de investigación: el manejo de la dimensión espacial de la información, particularmente si se trabaja localmente, lo que se puede lograr gracias a los SIG, que permiten visualizar simultáneamente diferentes capas de información sobre factores relacionados con los problemas de salud estudiados, asociados a un territorio específico, con todas las variables ambientales que implican, en un formato de lectura ágil y que hace evidente por sí mimo las relaciones y fenómenos.

\section{REFERENCIAS}

1. Castellanos P. Proyecto: sistemas nacionales de vigilancia de la situación de salud según condiciones de vida y del impacto de las acciones de salud y bienestar. Washington, D.C.: Organización Panamericana de la Salud; 1994. (PAHO/HDP/HDA/94.05).

2. Castellanos P. Perfiles de mortalidad, nivel de desarrollo e inequidades sociales en la región de las Américas. Washington, D.C.: Organización Panamericana de la Salud; 1994. (PAHO/ HDP/HDA/94.01).

3. Samaja J. La reproducción social y la relación entre la salud y condiciones de vida. Washington, D.C.: Organización Panamericana de la Salud; 1994. (PAHO/HDP/HDA/ 94.06).

4. Organización Panamericana de la Salud, Representación en Cuba; Grupo de Estudios Interdisciplinarios en Salud; Cuba, Ministerios de Salud. Sistema Nacional de Vigilancia de situación de salud según condiciones de vida. La Habana: OPS; 1994.

5. House J, Landis K, Umberson D. Social relationships and health. En: Kawachi I, Kennedy B, Wilkinson R, eds. The society and population health reader. I: Income inequality and health. New York: The New Press; 1999. Pp. 161-170.
6. Kawachi I, Kennedy B. Health and social cohesion: why care about income inequality? En: Kawachi I, Kennedy B, Wilkinson R, eds. The society and population health reader. I: Income inequality and health. New York: The New Press; 1999. Pp. 195-201.

7. Stansfeld S. Social support and social cohesion. En: Marmot M, Wilkinson R, eds. Social determinants of health. $2^{\mathrm{a}}$ ed. New York: Oxford University Press; 2000. Pp. 155-178.

8. Macintyre S, Ellaway A. Ecological approaches: rediscovering the role of the physical and social environment. En: Berkman L, Kawachi I, eds. Social epidemiology. New York: Oxford University Press; 2000. Pp. 332-348.

9. Alexander MJ, Laska E. La información como apoyo a los programas y servicios de salud mental en la comunidad. En: Levav I, ed. Temas de salud mental en la comunidad. Washington, D.C.: Organización Panamericana de la Salud. PALTEX; 1992. Pp. 78-99. (OPS. Serie PALTEX para ejecutores de Programas de Salud, 19).

10. Caraveo J. La vigilancia epidemiológica en el campo de la psiquiatría y la salud mental. Salud Mental 1985;8(2):20-22.
11. Gofin J, Levav I, Saraceno B. Vigilancia y evaluación en los programas de salud mental comunitaria. En: Levav I, ed. Temas de salud mental en la comunidad. Washington, D.C.: Organización Panamericana de la Salud. PALTEX; 1992. Pp 48-55. (OPS. Serie PALTEX para ejecutores de Programas de Salud, 19).

12. Teutsch S, Churchill R. Principles and practice of public health surveillance. New York: Oxford University Press; 1994.

13. American Psychiatric Association. Diagnostic and statistic manual of mental disorders. $4^{\text {a }}$ ed. Washington: The American Psychiatric Association; 1994.

14. Chile, Ministerio de Salud: Las enfermedades mentales en Chile: magnitud y consecuencias. Santiago de Chile: Ministerio de Salud; 1999.

Manuscrito recibido el 20 de junio de 2001. Aceptado para publicación, tras revisión, el 11 de diciembre de 2001. 
ABSTRACT Objective. To design and test a surveillance system to detect and rank the most significant mental health problems in a community and to establish their association with the living conditions in that community.

\section{Design and implementation of an epidemiological surveillance system for mental health}

Methods. The system was designed and tested at a primary health care center in the community of La Florida, which is in the metropolitan region of Santiago, the capital of Chile. The health problems that were under surveillance, with monthly monitoring, were: addiction (to alcohol, tobacco, and benzodiazepines or other drugs), violence (domestic violence and sexual abuse, real or suspected), and disorders in children (attention deficit and behavioral disorders). By means of a computerized geographical information system (GIS), those surveillance data were combined with other data. These other data included sociodemographic information on the persons who went to the health center for care as well as data on local risk factors and protective factors for health problems, some of which are indicators of the local residents' living conditions. Results. The proposed epidemiological surveillance system, which takes into account local living conditions and environmental variables, is a good tool for health action. The proposed system also complements the incomplete perspective of monitoring systems, which do not include variables describing the local context and that focus only on health problems. The information synthesized in the GIS maps makes it possible to simultaneously display different layers of information on factors related to the health problems studied, linked to a specific area and all its environmental variables, in an easy-to-read, self-explanatory format.

Conclusions. These results and the concurring opinions of the participating physicians show that the experimental system effectively met the basic requirements of an epidemiological surveillance system of this kind.

\section{First Puerto Rican Conference on Public Health}

Dates: $\quad$ 10-12 April 2002

Location: Caribe Hilton Hotel

San Juan, Puerto Rico

The First Puerto Rican Conference on Public Health will look at and critically assess the public health experience of Puerto Rico, looking at both the successes and the failures that the island has experienced. The conference is being organized by the Graduate School of Public Health of the University of Puerto Rico.

The conference will include plenary sessions by noted speakers, special sessions on key public health issues, and oral-presentation and poster-presentation sessions. The conference will be conducted in both English and Spanish. The registration fee for the conference (not including hotel accommodations and meals) is US\$ 175 (US\$ 50 for students) if paid by March 30, and US\$ 200 (US\$ 75 for students) if paid after that date.

Information (in English and Spanish):

First Puerto Rican Conference on Public Health

Graduate School of Public Health

Medical Sciences Campus

University of Puerto Rico

P.0. Box 365067

San Juan, Puerto Rico 00936-5067

United States of America

Telephone: (787) 758-2525, extension 1420

Fax: (787) 754-6621

E-mail: pcprsp@rcm.upr.edu

Internet: http://www.rcm.upr.edu/publichealth/conferencia2002.htm 